\title{
Long-term Trends in Annual Ground Snow Maxima for the Carpathian Region
}

\author{
Árpád Rózsás ${ }^{1,2, a^{*}}$, Miroslav Sýkora ${ }^{1, b}$, and László Gergely Vigh ${ }^{2, c}$ \\ ${ }^{1}$ Department of Structural Reliability, Klokner Institute, Czech Technical University in Prague, \\ Solinova 7, 16608 Prague, Czech Republic \\ ${ }^{2}$ Department of Structural Engineering, Budapest University of Technology and Economics, 3-9. \\ Müegyetem rkp., Kmf. 85, Budapest, H-1111, Hungary

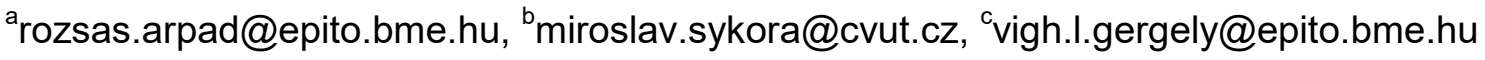 \\ * corresponding author
}

Keywords: ground snow, non-stationary, maximum likelihood, extreme value theory, structural reliability.

\begin{abstract}
The current structural design provisions are prevalently based on experience and on the assumption of stationary meteorological conditions. However, the observations of past decades and advanced climate models show that this assumption is debatable. Therefore, this paper examines the historical long-term trends in ground snow load maxima, and their effect on structural reliability. For this purpose, the Carpathian region is selected, and data from a joint research effort of nine countries of the region are used. Annual maxima snow water equivalents are taken, and univariate generalized extreme value distribution is adopted as a probabilistic model. Stationary and five nonstationary distributions are fitted to the observations utilizing the maximum likelihood method. Statistical and information theory based approaches are used to compare the models and to identify trends. Additionally, reliability analyses are performed on a simple structure to explore the practical significance of the trends. The calculations show decreasing trends in annual maxima for most of the region. Although statistically significant changes are detected at many locations, the practical significance - with respect to structural reliability - is considerable only for a few, and the effect is favourable. The results indicate that contrary to the widespread practice in extreme event modelling, the exclusive use of statistical techniques on the analysed extremes is insufficient to identify practically significant trends. This should be demonstrated using practically relevant examples, e.g. reliability of structures.
\end{abstract}

\section{Introduction}

Snow is an important climatic action, which governs the reliability of many structures, particularly lightweight steel structures [1]. These should operate without major structural maintenance for typically 50 years, and their real working life generally spans over 100 years. Therefore, it is of utmost importance that the expected actions are appropriately anticipated during the design process. The provisions of current structural standards are based on the assumption that the underlying natural phenomena - generating the actions on structures, and determining their working environment - are stationary. However, observations of the past decades and advanced meteorological analyses show that this assumption represent accurately neither the present, nor the future world [2]. Hence, the aim of this paper is to analyse the long-term trends in snow events and their implications on structural reliability. The Carpathian region is selected for the study, and observations from 1962 to 2011 are used to propose appropriate probabilistic models of snow maxima. Another database on which the online snow map for the Czech Republic [3,4] is based can be used in further studies for comparative purposes.

According to the authors' knowledge, the long-term trends in extreme snow loads, i.e. annual maxima, for the Carpathian region have not been sufficiently analysed yet. Similar research is 
available for the Swiss Alps, where Marty and Blanchet [5] detected statistically significant negative, long-term trends in annual snow depth. However, their analysis is focused on statistical significance testing and on return values. Thus, although indicating interesting tendencies, the practical significance of their results, e.g. with respect to structural reliability, should be analysed. This approach is prevalent in extreme value modelling, i.e. relying on significance testing, such as likelihood ratio test. In more detailed studies, the effect size [6] and confidence intervals are also used [5], but the practical significance that could support decision making is rarely investigated. Therefore, in this paper, a more pragmatic approach is promoted, in which after the statistical analysis the practical significance of the findings is examined. Main interest herein is the effect of snow trends on structural reliability, particularly on the probability of structural failure.

\section{Meteorological Data}

The meteorological data are obtained from the CarpatClim database, which is the outcome of research cooperation between nine countries of the Carpathian region [7]. The database provides snow water equivalent (SWE) values with $\sim 10 \mathrm{~km}$ spatial and daily temporal resolutions for a 19622011 period. These SWEs are used to develop the probabilistic models. Uncertainty due to measurement error and uncertainty of meteorological models used for processing the measurements are neglected. The reason is twofold: $(i)$ these uncertainties are hard to quantify and require further studies, and (ii) it is anticipated that these have minor effect on long-term trends.

\section{Statistical Analysis}

Climatic actions on structures are almost solely described by statistical distributions since the underlying natural phenomena are too complex to be represented by physical models. This fully probabilistic approach is adopted here as well, and the ground snow intensity is modelled by a single random variable. Univariate generalized extreme value (GEV) distribution, widely accepted in extreme value analysis is selected. The mathematical support is given by the extreme value theory, which states that independent, identically distributed block maxima will asymptotically converge to the generalized extreme value family, irrespectively of the parent distribution [8]. The adopted block length is one year, covering a whole winter season. Furthermore, to account for a season without snow, the cumulative distribution function of annual maxima is expressed as follows:

$$
P(X<x)=P(\text { snowfall }) \cdot P(X<x \mid \text { snowfall })
$$

where the former event has Bernoulli, while the latter has a GEV distribution.

Stationary Model. The standard parametrization of GEV distribution is used with shape ( $\xi$ ), scale $(\sigma)$ and location $(\mu)$ parameters [8]. The GEV distribution family comprises three special types: Weibull $(\xi<0)$, Gumbel $(\xi=0)$ and Fréchet $(\xi>0)$. Unlike the latter two, the first has an asymptotic upper bound.

Non-stationary Models. Five non-stationary models are considered, the time-dependency is introduced through the distribution parameters: location parameter with linear $\left(\mu_{1}\right)$ and quadratic $\left(\mu_{2}\right)$ function in time, while the other parameters are constant. The same parametrizations in respect of the scale parameter $\left(\sigma_{1}, \sigma_{2}\right)$, and a model with linear trend in both location and scale parameters $\left(\mu_{1} \sigma_{1}\right)$ while the shape parameter is constant, are adopted.

Parameter Estimation. Maximum likelihood parameter estimation (ML) technique is applied for a given distribution. The method provides values of parameters, under which the observations are most likely generated by the distribution. The parameter estimation uncertainty is taken into account by confidence intervals using the asymptotic normality property of the maximum likelihood estimator of the distribution parameters. For other, derived parameters the delta method is applied to construct confidence intervals [8]. 
Model Selection. Likelihood ratio (LR) test and Akaike information criterion (AIC) are used to compare the stationary and non-stationary models. The former is a standard frequentist hypothesis test for comparing nested models [9]. The latter is an asymptotic information criterion, which is based on the premise that the model with smallest information loss (Kullback-Leibler divergence) should be preferred. In the absence of the true model, the information loss cannot be calculated in absolute terms; however, the models can be compared and their relative 'strength' can be expressed by the difference in AICs or by using Akaike weights. In this paper the corrected form of Akaike information criterion (AICc) is applied, which takes into account the effect of a finite sample size. The Akaike weight of the $i^{\text {th }}$ model is then expressed as [9]:

$$
w_{i}=\exp \left(-1 / 2 \cdot\left(A I C c_{i}-A I C c_{\min }\right)\right) / \sum_{j=1}^{K} \exp \left(-1 / 2 \cdot\left(A I C c_{j}-A I C c_{\min }\right)\right)
$$

where $K$ is the number of models under consideration. The goodness-of-fit of the models at some locations are visually checked using Q-Q, P-P, and return period-return value plots [8]. Additionally, the GEV model is compared to other commonly applied distributions for diagnostic purposes, i.e. Gumbel and three-parameter lognormal. Due to the limitations of statistical significance testing [6] the effect size, power of the test, and confidence intervals are also utilized to compare the models. More importantly, the conclusions are based on the practical effect of these models on structural reliability.

\section{Results of Statistical Analyses}

Stationary Model. Stationary GEV distribution is fitted to each of the 5895 grid points using the ML method; the corresponding shape parameters are plotted in Fig. 1. The maps are created using equidistant conic projection and linear interpolation. In Fig. 1, the areas bounded by black contours indicate Weibull distribution $(\xi<0)$, it can be seen that the high mountainous areas have dominantly this upper bounded distribution type. Considerable part of the studied area has Fréchet distribution with relatively large shape parameter $(\xi>0.2)$, which is in conflict with the Gumbel assumption accepted in EN 1991-1-3:2003 and ISO 4355:2013. Additional limitation of the Gumbel distribution is that it yields to artificially narrower confidence intervals than the Fréchet as its parameters span smaller space [10-12].
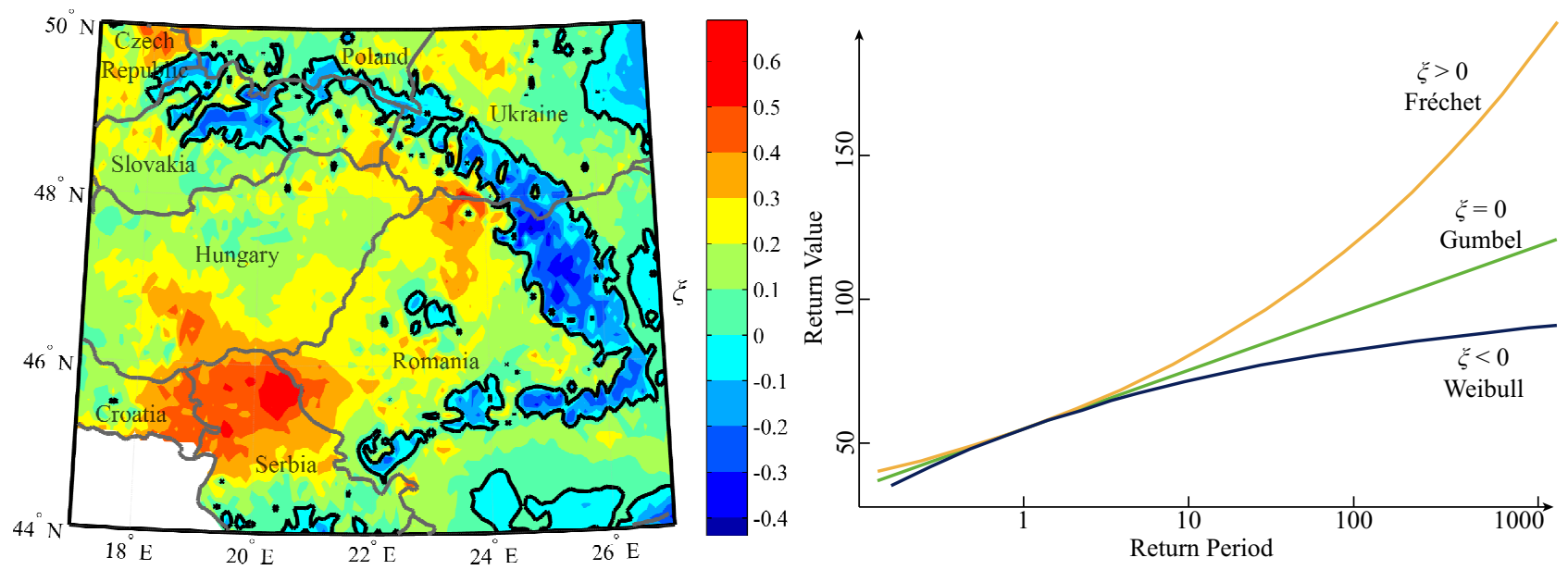

Fig. 1: Shape parameter $(\xi)$ of the fitted, stationary GEV distributions, the black contour surrounds the Weibull type (left), and illustration of GEV family types in Gumbel space (right). For interpretation of the references to colour in this figure legend, the reader is referred to the web version of this article.

The unfavourable deviation from Gumbel distribution is the most pronounced in the northern part of Serbia. It is interesting that some locations exhibit shape parameter larger than 0.5 , which means that the distribution has infinite variance. If this is deemed unrealistic, it might be avoided by adopting constraints in ML or by using Bayesian approaches with appropriate priors. 
Long-term Trends. First, a linear line is fitted to the annual maxima at every grid point in the leastsquares sense, while the snow-free years are discarded from the analysis. The associated slope parameters are illustrated in Fig. 2, where the negative values are referring to decreasing trends in time. At $97 \%$ of locations a decreasing trend is identified, whereas a mild increase is observed in the northern part of the region - Slovakia, the Czech Republic, and Poland. The small regions with strong, increasing trends next to decreasing ones (orange areas surrounded by dark blue ones) in Slovakia and Ukraine imply discrepancy in the database. Areas with the most pronounced decreasing trend are matching well with the Weibull regions, with the exception of the southern part. Based on the linear trend, the average change in annual maxima for the entire region, during observation period is $-42 \%$, compared to the 1962 level.
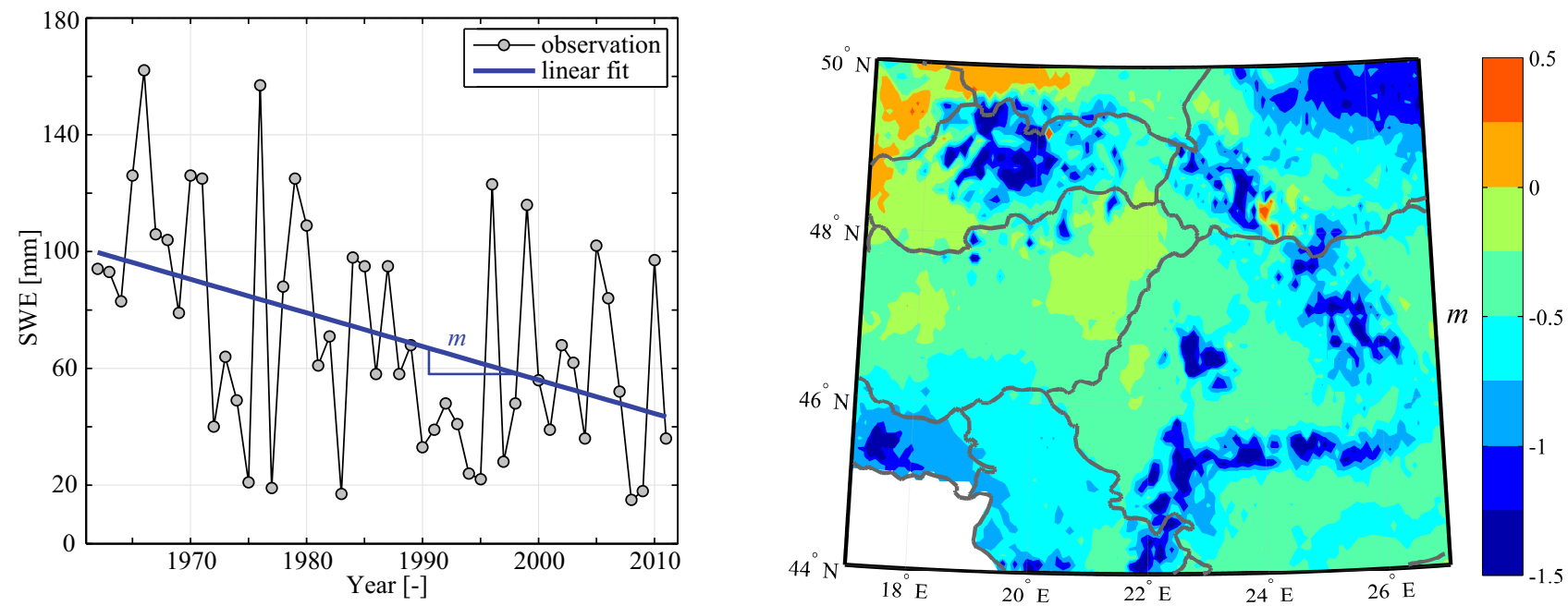

Fig. 2: A representative location with decreasing trend (left), and map of the linear trend line's slope parameter $m$ in $\mathrm{mm} /$ year (right). For interpretation of the references to colour in this figure legend, the reader is referred to the web version of this article.
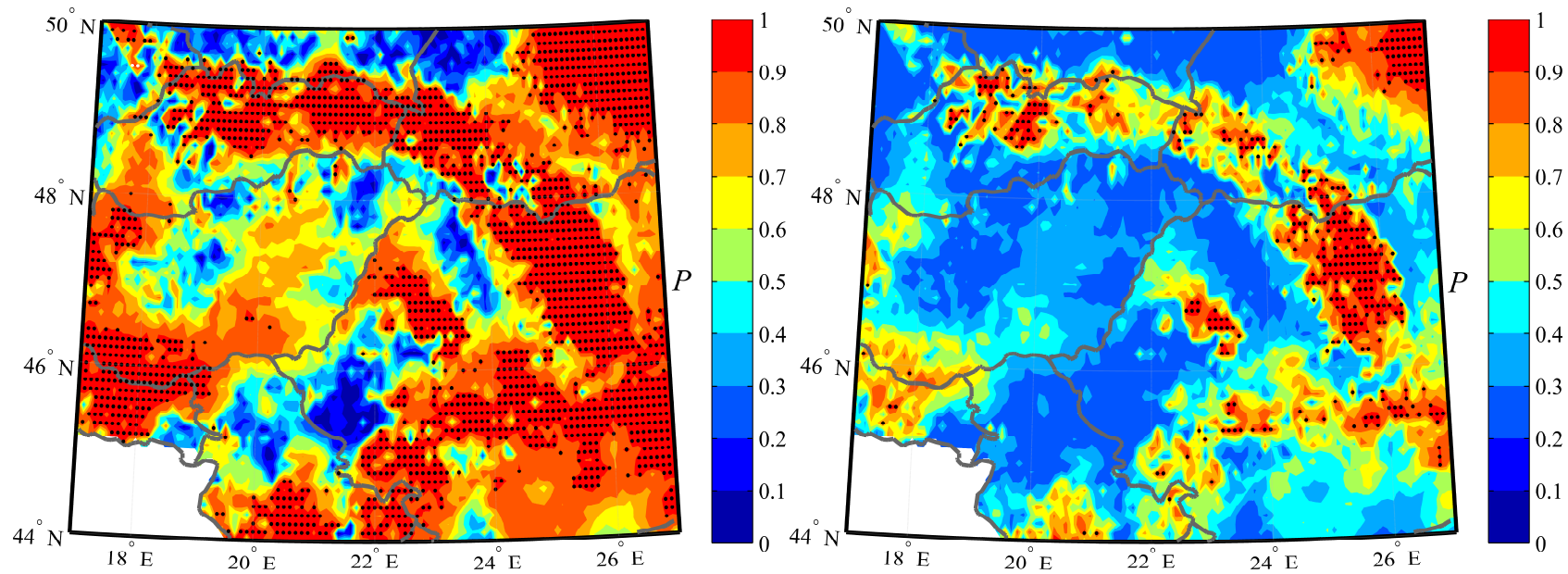

Fig. 3: Likelihood ratio (left) and Akaike weight (right) based comparison of $\mu_{1}$ and stationary models, grid-points with $P>0.90$ are marked with dots: black for decreasing and white for increasing trends. For interpretation of the references to colour in this figure legend, the reader is referred to the web version of this article.

Albeit both figures are showing probabilities $(P)$, their interpretation is substantially different. In case of the LR test it expresses the probability that if the null hypothesis is true (stationary model) the difference between the loglikelihoods is at least as large as the one observed. This probability is typically referred as a $p$-value, and the left side of Fig. 3 is showing the complementary probability, $P=1-p$. On the other hand, the Akaike weights - illustrated on the right side of Fig. 3 - express the probability that $\mu_{1}$ model is better than the stationary one, in the Kullback-Leibler divergence sense [9]. In respect of these probabilities, in both cases the selected threshold is $90 \%$, locations above this value are marked with a dot and considered as statistically significant. The vast majority of the statistically significant trends identified by the LR test are decreasing (black dots), only two 
locations in the Czech Republic show significant increase (white dots in the upper left corner of Fig.3 left). The Akaike weight based comparison shows solely decreasing trends. The LR test identified much more locations with a significant trend, although the probabilities are not directly comparable. In addition, $65 \%$ of these locations are statistically significant even with the $95 \%$ threshold level. The trends might be explained by the diminishing number of snow days due to an increasing mean global temperature.

A more pragmatic comparison of time trends is presented in Fig. 4, where the 50-year return values for stationary and non-stationary $\mu_{1}$ models are compared. The 50-year return value is referred as a characteristic value in structural engineering and serves as the basis of everyday design. All of the selected locations, shown in Fig. 4, exhibit a significant negative trend in AICc sense.

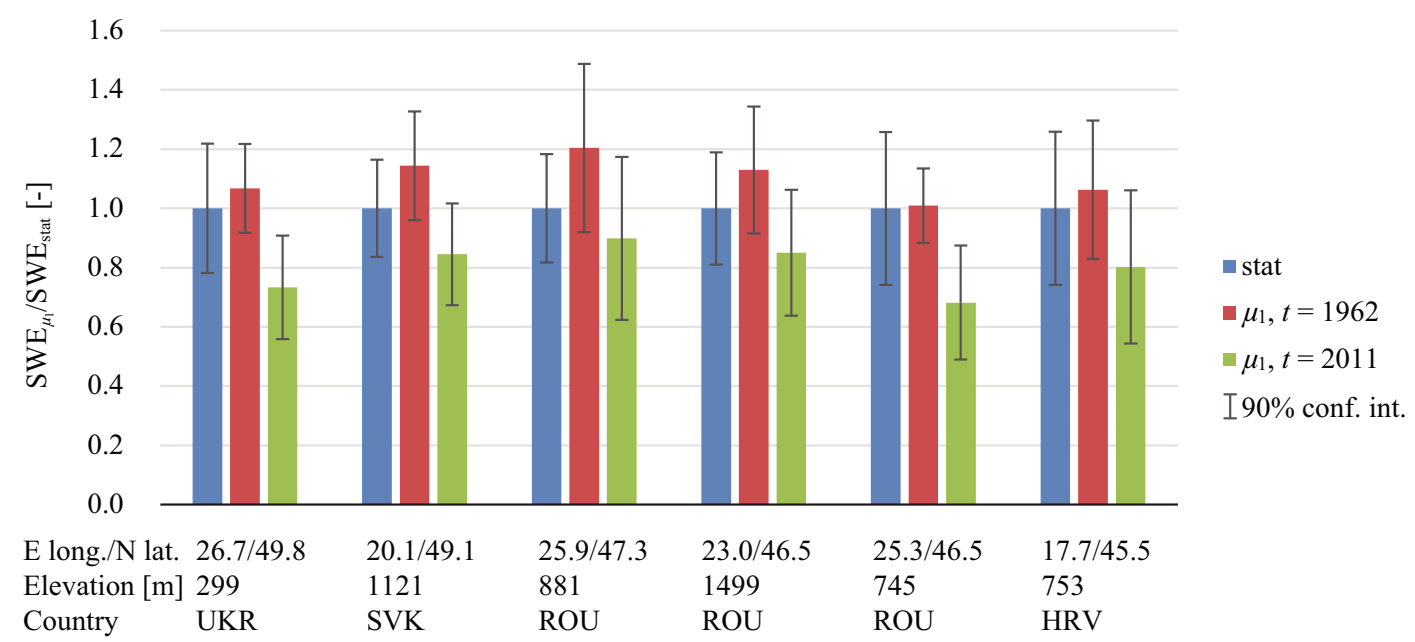

Fig. 4: Normalized 50-year return value SWE with 90\% confidence intervals for selected locations, with significant negative trends as identified by Akaike weights.

Fig. 4 also shows that the difference is often practically insignificant even for the locations where the AICc based criterion identified significant trends (in respect of 50-year return value with $90 \%$ confidence interval). The time trend is deemed practically significant if the stationary point estimate is outside of the non-stationary $90 \%$ confidence interval. For the entire region, using $\mu_{1}$ model the average change in respect of characteristic values between 1962 and 2011 is -10\%, using year 1962 as reference. For locations with statistically significant trends the changes are $-19 \%$ and $-26 \%$ for LR test and Akaike weights respectively.

\section{Effect on Structural Reliability}

Although the focus of this paper is the statistical characterization of snow loads and identification of trends in annual maxima, the key question from an engineering point of view is the effect of the changes on structural reliability and the adequacy of current provisions. The simple statistical, information theory (LR, AICc), and representative quantile based comparisons cannot answer this question, since the failure probability of a structure is dominantly determined by the tail of the distribution functions, well above or below the characteristic value. Thus, a simple, illustrative example is selected to explore this question. The related limit state function and the properties of the random variables are given in Eq. (3) and in Table 1, respectively. It approximately represents a lightweight steel structure with governing snow load. For simplicity, the distribution function of annual snow maxima is used and annual failure probabilities are calculated. Exceptional snow loads and accidental load combinations are not covered in the analysis.

$$
g=R-(G+\mu \cdot S \cdot 0.981)
$$

Reliability analyses are performed for two representative locations using standard, first order reliability analysis. The first one is the Ukrainian location presented in Fig. 4, it has small, scattered settlements and it represents the significant negative trend even in respect of 50-year return values. 
The second is the fifth most populous city in Hungary (Pécs), it is identified by the LR test with statistically significant negative trend, and it represents the moderate negative trend, which reflects well the majority of the region. These two locations are referred hereinafter as Ukrainian and Hungarian. Reliability is analysed considering a reference period of one year. The location parameter is obtained from the non-stationary model for each of the selected years - 1962, 2011 and 2062. The delta method is used to construct approximate confidence intervals for these indices, and the results are summarized in Fig. 5.

Table 1: Properties of random variables in the limit state function.

\begin{tabular}{lllll}
\hline Variable & Distr. type & Mean & CoV & Ref. \\
\hline Resistance, $R[-]$ & Lognormal & $1000 / 350^{\dagger}$ & 0.12 & {$[13]$} \\
Ground snow, $S[-]$ & GEV & $37 / 72^{\dagger}$ & $0.78 / 0.54^{\dagger *}$ & - \\
Ground to roof conversion factor, $\mu[-]$ & Lognormal & 0.75 & 0.15 & {$[14]$} \\
Permanent action, $G[-]$ & Normal & 50 & 0.10 & {$[14]$} \\
\hline
\end{tabular}

* from unbiased moments of the observations.

${ }^{\dagger}$ data to Hungarian/Ukrainian locations.



Fig. 5: Reliability indices with 0.75 confidence intervals from ground snow parameter estimation uncertainty.

From Fig. 5 it is clear that the moderate decreasing trend has practically no effect on structural reliability that is dominated by the parameter estimation uncertainty. For the Ukrainian location the increase in reliability level is practically significant, although the uncertainty is large here as well. From a safety point of view, the change is clearly favourable, and whether the current provisions will provide economical design for these locations in the future is a different issue. The most important factor in the difference of the results for the Hungarian and Ukrainian locations is that the former has Fréchet, while the latter has Weibull distribution.

The extrapolation of the non-stationary model to 2062 should be handled with care, it is provided only for illustrative purposes. It introduces additional uncertainty, as illustrated for the Ukrainian location in Fig. 6. This should be reduced by use of physical models or further explanations.

The results are dependent on the number of observations and on the reference period used in the reliability analyses. More data would yield to narrower confidence intervals and allow sharper, stronger conclusions, while an extended reference period would level out the differences in annual failure probabilities.

\section{Summary and Conclusions}

This paper analyses the long-term trends in extreme snow loads for the Carpathian region and their effect on structural reliability. Annual snow water equivalent maxima are used, which are collected during the 1962-2011 period. Univariate generalized extreme value distribution is fitted to the data applying the maximum likelihood method. A stationary and five non-stationary generalized extreme value models are used to describe the observations. Likelihood ratio test and Akaike 
weights, supplemented with reliability analyses are used to evaluate the significance of trends. The main conclusions of this study are summarised as follows:

- For the majority of lowland and highland locations Fréchet distribution seems to be appropriate while Weibull distribution fits the data better for mountains.

- A decreasing trend in annual snow maxima is found for the $97 \%$ of the studied region.

- The likelihood ratio test identified numerous locations with statistically significant $(p<0.10)$ decreasing trends. However, the power of the test in average is low, and the effect size compared to confidence intervals regarding the 50-year return value reveals substantial uncertainty.

- The likelihood ratio test and Akaike weights suggest that the trend in the annual maxima can be better captured by allowing trend in the location parameter than in the scale parameter.

- The reliability analyses show that the practical significance of time trends not necessary follows the statistical significance. This is largely due to the substantial uncertainty in the parameter estimation.

- The reliability analyses indicate that for most locations in the region, which are characterized by Fréchet distribution, the negative trend in annual snow maxima has a minor effect on structural reliability, the uncertainty in parameter estimation is governing. Moreover, it is deemed unjustified to extrapolate trends based on about 50 years to thousands of years that represent a relevant return periods for the Ultimate Limit States.

- For locations with a strongly decreasing trend and Weibull distribution, the effect of the trend on structural reliability is practically significant, although the change is favourable from a safety point of view.

The presented approach can be applied for other basic variables significantly affecting structural reliability such as other variable loads or resistance parameters. A limitation of this study is that only generalized extreme value distribution is considered. This can have an important effect on the failure probability that is governed by the tail of the distribution, i.e. very rare, not even observed events. This effect could be investigated by more involved statistical techniques, but only by means of additional data or by accurate physical models could be overcome, which are rarely available.



Fig. 6: Ukrainian location, 50-year return value SWE $\left(q_{50}\right)$ with $90 \%$ confidence bands (CI90\%) for stationary (stat) and $\mu_{1}$ models.

\section{Acknowledgement.}

This work was partly supported by the International Visegrad Fund Intra-Visegrad Scholarship (contract no. 51401089), by the Czech Science Foundation (project no. P105/12/G059), by the Czech Ministry of Education, Youth and Sports (project no. LG14012), and by the János Bolyai Research Scholarship of the Hungarian Academy of Sciences. The meteorological data were obtained from the CARPATCLIM Database (C) European Commission - JRC, 2013. The calculations are completed using Matlab 2014a, FERUM 4.1 (Finite Element Reliability Using Matlab), and R 2014 (A language and environment for statistical computing), the work and commitment of the developers of these applications are highly appreciated. All of the related codes/scripts, processed data and results are available from the corresponding author. 


\section{References}

[1] J. Geis, K. Strobel, A. Liel, Snow-Induced Building Failures, Journal of Performance of Constructed Facilities, 26(4) (2011) 377-388, doi: 10.1061/(asce)cf.1943-5509.0000222.

[2] IPCC, Managing the Risks of Extreme Events and Disasters to Advance Climate Change Adaptation, In C. B. Field, V. Barros, T. F. Stocker, D. Qin, D. J. Dokken, K. L. Ebi, M. D. Mastrandrea, K. J. Mach, G.-K. Plattner, S. K. Allen, M. Tignor, P. M. Midgley (Eds.), A Special Report of Working Groups I and II of the Intergovernmental Panel on Climate Change, Cambridge, UK, and New York, NY, USA, 2012.

[3] Křivý, V., Brož, P., Němec, L., Studničková, M, The Usage of Digital Map of Snow Load on the Ground for the Area of the Czech Republic (in Czech), Casopis stavebnictví, 2011, v. 10, No. 1112/2011, p. 56-58, ISSN 1802-2030.

[4] Křivý, V., Čajka, R, Design and reliability assessment of roof structural elements using the new digital ground snow load map of the Czech Republic, In Proceedings of 17th International conference Engineering Mechanics 2011, Svratka : Academy of Sciences of the Czech Republic, 2011, ISBN 978-80-87012-33-8.

[5] C. Marty, J. Blanchet, Long-term changes in annual maximum snow depth and snowfall in Switzerland based on extreme value statistics, Climatic Change, 111(3-4) (2012) 705-721, doi: 10.1007/s10584-011-0159-9.

[6] R.P. Carver, The Case Against Statistical Significance Testing, Revisited, The Journal of Experimental Education, 61(4) (1993) 287-292, doi: 10.1080/00220973.1993.10806591.

[7] S. Szalai, I. Auer, J. Hiebl, J. Milkovich, T. Radim, P. Stepanek, P. Zahradnicek, Z. Bihari, M. Lakatos, T. Szentimrey, D. Limanowka, P. Kilar, S. Cheval, G. Deak, D. Mihic, I. Antolovic, V. Mihajlovic, P. Nejedlik, P. Stastny, K. Mikulova, I. Nabyvanets, O. Skyryk, S. Krakovskaya, J. Vogt, T. Antofie, J. Spinoni, Climate of the Greater Carpathian Region Final Technical Report, 2013, www.carpatclim-eu.org.

[8] S. Coles, An Introduction to Statistical Modeling of Extreme Values, Springer-Verlag, London, 2001.

[9] K.P. Burnham, D.R. Anderson, Model Selection and Multimodel Inference, A Practical Information-Theoretic Approach, second ed., Springer-Verlag, New York, 2002.

[10] S. Coles, L. Pericchi, Anticipating catastrophes through extreme value modelling, Journal of the Royal Statistical Society, Series C (Applied Statistics), 52(4) (2003) 405-416.

[11] Á. Rózsás and M. Sýkora, Model Comparison and Quantification of Statistical Uncertainties for Annual Maxima of Ground Snow Loads, In L. Podofillini, B. Sudret, B. Stojadinovic, E. Zio and W. Kröger (eds.), Safety and Reliability of Complex Engineered Systems - Proceedings of the European Safety and Reliability Conference ESREL 2015, Zurich, 7-10 September 2015. Leiden: Balkema/CRC Press, p. 2667-2674, 2015, ISBN 978-1-138-02879-1 (Hbk + CD-ROM), 978-1-31564841-5 (eBook PDF).

[12] Á. Rózsás and M. Sýkora. Effect of Statistical Uncertainties in Ground Snow Load on Structural Reliability (accepted for publication), In Proceedings of IABSE Geneva Conference 2015, 23-25 September 2015.

[13] JCSS, JCSS Probabilistic Model Code Part III - Resistance Models: Joint Committee on Structural Safety, 2000.

[14] JCSS, JCSS Probabilistic Model Code Part II - Load Models: Joint Committee on Structural Safety, 2001. 